\title{
Fully Connected Object Proposals For Video Segmentation
}

\section{Conference Paper}

Author(s):

Perazzi, Federico; Wang, Oliver; Gross, Markus; Sorkine-Hornung, Alexander

Publication date:

2015

Permanent link:

https://doi.org/10.3929/ethz-a-010566328

Rights / license:

In Copyright - Non-Commercial Use Permitted

Originally published in:

https://doi.org/10.1109/ICCV.2015.369 


\title{
Fully Connected Object Proposals for Video Segmentation
}

\author{
Federico Perazzi $^{1,2} \quad$ Oliver Wang $^{2} \quad$ Markus Gross $^{1,2} \quad$ Alexander Sorkine-Hornung ${ }^{2}$ \\ ${ }^{1}$ ETH Zurich $\quad{ }^{2}$ Disney Research Zurich
}

\begin{abstract}
We present a novel approach to video segmentation using multiple object proposals. The problem is formulated as a minimization of a novel energy function defined over a fully connected graph of object proposals. Our model combines appearance with long-range point tracks, which is key to ensure robustness with respect to fast motion and occlusions over longer video sequences. As opposed to previous approaches based on object proposals, we do not seek the best per-frame object hypotheses to perform the segmentation. Instead, we combine multiple, potentially imperfect proposals to improve overall segmentation accuracy and ensure robustness to outliers. Overall, the basic algorithm consists of three steps. First, we generate a very large number of object proposals for each video frame using existing techniques. Next, we perform an SVM-based pruning step to retain only high quality proposals with sufficiently discriminative power. Finally, we determine the fore- and background classification by solving for the maximum a posteriori of a fully connected conditional random field, defined using our novel energy function. Experimental results on a well established dataset demonstrate that our method compares favorably to several recent state-of-the-art approaches.
\end{abstract}

\section{Introduction}

Video object segmentation refers to the partitioning of a video into two disjoint sets of pixels representing a foreground object and background regions. The abundance of available literature on video segmentation reflects the importance of the topic, which is an essential building block for numerous applications including video editing and postprocessing, video retrieval, analysis of large video collections, activity recognition, and many more. Many existing approaches are based on background subtraction, tracking of feature points and homogeneous regions, spatiotemporal graph-cuts, or hierarchical clustering. Recently, methods leveraging advances in object recognition have gained popularity. These methods make use of per-frame object proposals and employ different techniques to select a set of temporally coherent segments, one per frame, typically by
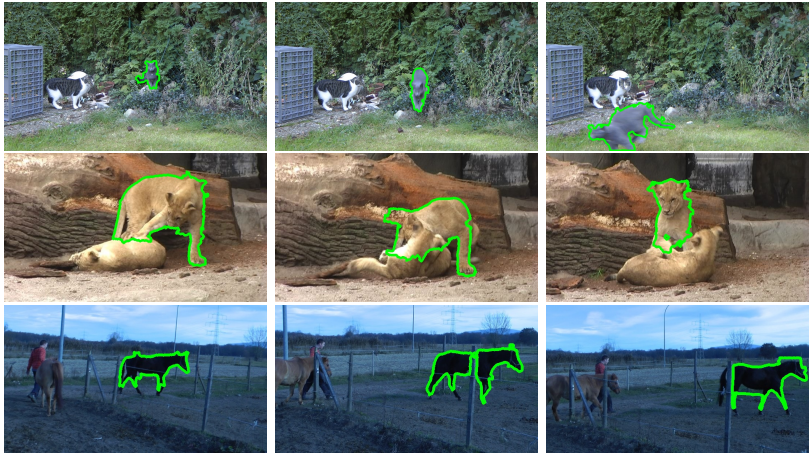

Figure 1. Example segmentations using our approach on three sequences of the Freiburg-Berkeley Motion Segmentation Dataset [5]. Top to bottom: we demonstrate robustness to challenging situations typical of unconstrained videos such as fastmotion and motion blur, color ambiguities between fore- and background, and partial occlusions.

minimizing an energy function defined over a locally connected spatiotemporal graph. While these methods have achieved state-of-art performance [27, 30, 44], the sparse graph structure limits their ability to segment videos with fast motion and occlusions.

We propose an efficient alternative approach which exploits a fully connected spatiotemporal graph built over object proposals. We map our similarity term into a Euclidian space, which is computationally efficient to optimize and well suited for modeling long-range connections. The fully connected nature of the graph implies information exchange between both spatially and temporally distant object proposals, which in turn enables our method to be robust to the difficult cases of fast frame-to-frame motion and object occlusions. We additionally propose an energy term that incorporates sparse but confident long range feature tracks, in order to ensure similar temporal labeling of objects. While previous approaches are constrained to the selection of one proposal per frame, our formulation enables the grouping of multiple overlapping proposals in the same frame, yielding robustness to outliers and incorrect proposal boundaries.

Given as input a set of object proposals and a few annotated foreground proposals, our algorithm consists of three steps. Initially, a rough classification and subsampling of 
the data is performed using a self-trained Support Vector Machine (SVM) classifier in order to reduce the size of the proposal space while preserving a large pool of candidate foreground proposals. Next, maximum a posteriori (MAP) inference is performed on a fully connected conditional random field (CRF) to determine the final labeling of the candidate proposals. Finally, each labeled proposal casts a vote to all pixels that it overlaps. The aggregate result yields the final foreground-background segmentation. We compare our results with an existing benchmark dataset and show that our method outperforms several state-of-theart approaches.

\section{Related Work}

In this section we provide an overview of commonly employed approaches and discuss works most related to ours.

Background Subtraction. Many established video segmentation algorithms are based on background subtraction. These approaches assume that the background is known a priori, and that the camera is stationary $[12,14]$ or undergoes a predictable, parametric motion [20,22,38]. While this family of methods cannot handle non-rigid camera movements, they are well suited to specific application scenarios such as surveillance systems [6]. In contrast our method is designed to handle unconstrained videos with arbitrary camera motion and non-rigid background.

Tracking-based Methods. Significant progress has been achieved by methods designed to track keypoints over time and, more recently, over image regions [4, 27, 41]. These methods, however, only consider two consecutive frames of video and cannot handle sudden motion and appearance changes (i.e. due to lighting). Related to tracking systems, Brox et al. propose an approach to segment motion by spectral clustering of long term point trajectories based on their motion affinity [5] and a variational approach [31] to turn the resulting sparse trajectory clusters into dense regions. By defining the pairwise distance between trajectories as the maximum difference of their motion, they assume a translational motion model. Despite this being a reasonable approximation for spatially close point trajectories, these methods have difficulties to segment articulated bodies following non-rigid motion. In this work, we exploit point-tracks to increase stability for long term temporal connections. However, we avoid motion clustering and do not make any assumption on the underlying object motion.

Oversegmentation. Unconstrained motion can be handled by supervoxel based methods $[19,21,43]$. These methods generate an oversegmentation of the video into spacetime homogeneous, perceptually distinct regions. They are important for early stage video preprocessing, but do not directly solve the problem of video object segmentation as they do not provide any principled approach to flatten the hierarchical decomposition of the video into a binary segmen- tation [32]. In Section 6 we formulate the problem of reducing overlapping segments into a foreground-background partition by minimizing a novel energy function which we solve optimally by inference on a CRF.

Video Object Segmentation. Recently, closely related to our work, a family of methods for solving the problem of segmenting the dominant moving object in a video gained popularity. Papazouglo and Ferrari [32] describe a fully automatic approach that efficiently identifies closed motion boundaries to determine the object position, and propagates the labeling through a spatiotemporal extension of GrabCut [39]. Ramakanth and Babu [37] extend work on videoretargeting [40] to propagate labels through connected lowenergy paths. Despite its ability to accurately segment object boundaries, this method cannot handle complete occlusions and fast motion, as it operates locally. To overcome this problem [17] propose a non-local consensus voting scheme defined over a globally connected graph. This method shares similarities with ours (fully connected graph, weighted voting), but differs as their graph is built over nonoverlapping pixel regions and they use a random-walk transition matrix to update their prediction iteratively until convergence. Relying on saliency detection this method might encounter difficulties to segment complex objects of multiple colors. To overcome this problem, Banica et al. [2] link figure-ground hypotheses instead of superpixels, to form temporal chains representing the object, based on saliency metrics and appearance similarity.

Proposal-based approaches. Recent advances in stateof-the-art image analysis $[8,18,28]$ have motivated the use of object proposals $[1,9,16,24]$ in video object segmentation. Lee et al. [26] discover clusters of key-segments in videos, coupling the notion of objectness and appearance similarity. Hypotheses are later ranked and the top scoring one is automatically selected for video segmentation. Their work is well suited to determine groups of segments with consistent appearance and motion, but disregards spatial and temporal relations between segments. $\mathrm{Ma}$ and Latecky [30] account for these by imposing the selection of one proposal in every frame, formulating the problem as finding a maximum weighted clique in a locally connected graph with mutex constraints. However, the strict assumptions that the object should appear in every frame limits their efficacy in real world scenarios. Similar to [30], Zhang et al. [44] create a layered Directed Acyclic Graph (DAG) which combines unary edges measuring the objectness of the object proposal and pairwise edges modeling affinities. A shortest path determines the video object segmentation. Both [30,44] formulate the problem on a locally connected graph structure, requiring that objects appear in every frame. In contrast, we exploit a fully connected graph to robustly model long range relations required to handle fast motion or occlusions. 


\section{Overview}

Our method consists of three stages. Given an input video $\mathcal{V}$, for each frame $\mathcal{V}^{t}$ we compute a large set of object proposals $\mathcal{S}^{t}=\left\{s_{i}^{t}\right\}$, using existing techniques [24]. The goal of this step is to generate a wide range of different proposals, such that a sufficient number of segments overlap with the object (Section 4). Then our method learns an SVM-based classifier in order to resample $\mathcal{S}$ into a smaller set of higher quality proposals $\overline{\mathcal{S}}$ (Section 5 ). Finally we refine this classification by solving for the maximum a posteriori inference on a densely connected CRF (Section 6). The fully connected graph structure is coupled with a novel energy function that considers overlap between point-tracks in the pairwise potentials, exploits temporal information, and ensures robustness to fast motion and occlusions.

\section{Object Proposal Generation}

Algorithms for computing object proposals are generally designed to have a high recall, proposing at least one region for as many objects in the image as possible. While the set of candidates must remain of limited size, the task of selecting positive samples is left to later stages, and the ratio of regions that truly belong to an object, i.e. precision, is usually not considered a measure of performance.

While other approaches leverage the high recall property by assuming that there is one good proposal per-frame, our goal is to exploit the redundancy in the data of multiple proposals with a high degree of overlap with the foreground object. In order to have a significant amount of such positive instances, we modified the parameters of [24] that control seed placement and level set selection to generate around twenty thousands proposals per frame. Otherwise we consider the proposal generator as a black box, and other object proposal methods could be used instead.

It is important to note, however, that the resulting set of proposals is likely imbalanced, with potentially many more proposals on background regions than on foreground, depending on object size. Furthermore, many proposals will cover both foreground and background. These issues negatively impact segmentation, both in terms of quality and efficiency. To overcome this problem we self-train an SVM classifier and resample the pool of proposals.

\section{Candidate Proposal Pruning}

We introduce a per-frame pruning step with the goal of rebalancing the set of proposals and selecting only those with higher discriminative power, i.e. those that do not overlap both with foreground and background. The choice of the SVM is justified by its proven robustness to skewed vector spaces resulting from class imbalance [42] and relatively fast performance. We train an SVM classifier which operates on elements of $\mathcal{S}$, separating those that overlap with foreground from those that belong to the background (Section 5.1), and then resample the set (Section 5.2). Finally, we use the output of the SVM to initialize the unaries of the CRF (Section 6.1).

\subsection{Feature Extraction and Training}

Features. From each of the proposals we extract a set of features that characterize its appearance, motion and objectness as summarized in Table 1. The global appearance and spatial support are defined in terms of average color, average position and area. The local appearance is encoded with Histogram of Oriented Gradients (HOG) [29] computed over the proposal bounding box rescaled to $64 \times 64$ pixels and divided into $8 \times 8,50 \%$ overlapping cells quantized into 9 bins. The motion is defined with Histogram of Oriented Optical Flow (HOOF) [10] extracted from the proposal bounding box also rescaled to $64 \times 64$ pixels and quantized into 32 bins. The objectness is measured in terms of region boundaries encoded by $8 \times 8$ normalized gradients patches [11]. The set of features is aggregated into a 1398 dimensional descriptor $\mathbf{x}_{i} \in \mathcal{X}$.

Training. The classifier is trained from a small set of proposals $\tilde{\mathcal{S}}$ known to belong to the foreground object. This set $\tilde{\mathcal{S}}=\left\{\tilde{s}_{i}\right\}$ may be either determined using automatic approaches such as salient object detectors [34], based on objectness [16, 26], manually using interactive video editing tools, or a combination thereof. In our experiments we manually annotated 1 or 2 foreground proposals persequence. $\tilde{\mathcal{S}}$ is augmented with all proposals that spatially overlap with one of its initial elements by a factor of more than a threshold $\tau$ (0.95 in our experiments). All remaining proposals are marked background. A binary SVM classifier with linear kernel and soft margins is trained on the labeled data yielding the score function $\mathcal{C}\left(\mathbf{x}_{i}\right)=\mathbf{w}^{T} \mathbf{x}_{i}+b$ which measures the distance of the proposal $\tilde{s}_{i}$ with associated feature vector $\mathbf{x}_{i}$ from the decision surface $\mathbf{w}_{\perp}$. While $\operatorname{sign}\left(\mathcal{C}\left(\mathbf{x}_{i}\right)\right)$ is enough to classify proposals as either foreor background, in Section 6 we can additionally include the distance from the hyperplane $\mathbf{w}^{T} \mathbf{x}_{i}+b \in[-\infty,+\infty]$ as the posterior probability $P\left(y_{i} \mid \mathbf{x}_{i}\right) \in[0,1]$ in order to initialize the unary potentials of the CRF. We use Platt Scaling [35] to fit a logistic regressor $\mathcal{Q}$ to the output of the SVM and the true class labels, such that $\mathcal{Q}\left(\mathcal{C}\left(\mathbf{x}_{i}\right)\right): \mathbb{R} \rightarrow P\left(y_{i} \mid \mathbf{x}_{i}\right)$. Parameters of the SVM are reported in Section 7.

\begin{tabular}{lll}
\hline Feature & Description & Dim \\
\hline (ACC) & Area, centroid, average color & 6 \\
(HOOF) & Histogram of Oriented Optical Flow & 32 \\
(NG) & Objectness via normalized gradients & 64 \\
(HOG) & Histogram of oriented gradients & 1296 \\
\hline
\end{tabular}

Table 1. Set of features extracted from each object proposal by the SVM classifier and corresponding dimensionality. 


\subsection{Classification and Resampling}

Given the trained classifier $\mathcal{C}$, we aim to roughly subdivide the set of object proposals $\mathcal{S}^{t}$ extracted at frame $t$ into two spatially disjoint sets $\mathcal{S}_{+}^{t}$ and $\mathcal{S}_{-}^{t}$ such that $\bigcup \mathcal{S}_{+}^{t}$ lies within the foreground region and $\bigcup \mathcal{S}_{-}^{t}$ on the background. Initially we form $\mathcal{S}_{+}^{t}=\left\{s_{i}^{t} \mid P\left(y_{i} \mid \mathbf{x}_{i}\right)>0.5\right\}$. Next, we select elements from the set of proposals classified as background such that they do not overlap with $\mathcal{S}_{+}^{t}$, i.e., $\mathcal{S}_{-}^{t}=\left\{s_{i}^{t}|| \mathcal{S}_{+}^{t} \cap s_{i}^{t} \mid<\bar{\epsilon}\right\}$. The slack variable $\bar{\epsilon}$ is necessary to avoid $\mathcal{S}_{-}^{t}=\emptyset$, which can happen in videos where the foreground object occupies most of the frame. We initially set $\bar{\epsilon}$ to 0 and iteratively increment it with steps of 20 until the constraint $\left|\mathcal{S}_{-}^{t}\right|>500$ is satisfied or the total amount of background proposal is reached. In our experiments we retain $\sim 10 \%$ of the proposals generated (roughly 2000 proposals per-frame).

The positive impact of our pruning and resampling step on the quality of the video segmentation is shown in Section 8. The resulting classification can still be imprecise, but serves the purpose of rebalancing positive and negative instances. The union of the two newly generated sets $\overline{\mathcal{S}}^{t}=\mathcal{S}_{+}^{t} \cup \mathcal{S}_{-}^{t}$ forms the input $\overline{\mathcal{S}}=\left\{\overline{\mathcal{S}}^{t}\right\}$ to the following step, which then provides a global solution considering spatial and temporal information jointly with the color appearance. Note that for ease of notation we refer to $\overline{\mathcal{S}}$ as $\mathcal{S}$ throughout the remaining part of paper.

\section{Fully Connected Proposal Labeling}

In order to accurately classify elements of $\mathcal{S}$, we must enforce a smoothness prior that says that similar proposals should be similarly classified. Conditional random fields provide a natural framework to incorporate all mutual spatiotemporal relationships between proposals as well as our initial proposal confidences.

\subsection{Inference}

Let us define a set of labels $\mathcal{L}=\{b g=0, f g=1\}$, corresponding to background and foreground regions respectively. Let $\mathcal{F}=\left\{\mathbf{f}_{i}\right\}$ be a newly generated set of features extracted from each element in $\mathcal{S}$, as defined in Eq. (3). Let us define the set of random variables $Y=\left\{y_{i}\right\}, y_{i} \in \mathcal{L}$. Consider a fully-connected random field $(Y, \mathcal{X} \cup \mathcal{F})$ defined over a graph $\mathcal{G}=(\mathcal{V}, \mathcal{E})$ whose nodes correspond to object proposals. Let $Z(\mathcal{X}, \mathcal{F})$ be the partition function. The posterior probability for this model is $P(Y \mid \mathcal{X}, \mathcal{F})=$ $\frac{1}{Z(\mathcal{F})} \exp (-\mathrm{E}(Y \mid \mathcal{X}, \mathcal{F}))$ with the corresponding Gibbs energy defined over the set of all unary and pairwise cliques:

$$
E(Y \mid \mathcal{X}, \mathcal{F})=\sum_{i \in \mathcal{V}} \psi_{u}\left(y_{i} ; \mathcal{X}\right)+\sum_{i, j \in \mathcal{E}} \psi_{p}\left(y_{i}, y_{j} ; \mathcal{F}\right) .
$$

Unary Potentials. The unary term $\psi_{u}$ is directly inferred from the output of the SVM and the set of annotated pro- posals $\tilde{\mathcal{S}}$. We formulate an updated conditional probability $P\left(y_{i} \mid \mathbf{x}_{i}\right)=\lambda \cdot \mathcal{Q}\left(\mathcal{C}\left(\mathbf{x}_{i}\right)\right)+\frac{(1-\lambda)}{2}$, with the user-defined parameter $\lambda \in[0,1]$ modulating the influence of the SVM prediction on the CRF initialization. For all experiments, we set the parameter $\lambda$ to 0.1 . We define $\psi_{u}$ as a piecewise function

$$
e^{-\psi_{u}\left(y_{i}, \mathcal{X}\right)}=\left\{\begin{array}{ll}
l_{i}+\hat{\epsilon}, l_{i} \in \mathcal{L} & s_{i} \in \tilde{\mathcal{S}} \\
P\left(y_{i} \mid \mathbf{x}_{i}\right) & s_{i} \notin \tilde{\mathcal{S}}
\end{array} .\right.
$$

Pairwise Potentials. We define the label compatibility function $\mu$ to be the Potts model $\mu\left(y_{i}, y_{j}\right)=\left[y_{i} \neq\right.$ $\left.y_{j}\right]$, a Gaussian kernel $k_{*}(x)=\exp \left(-\frac{x^{2}}{2 \sigma_{*}^{2}}\right)$, and scalar weights $\omega_{*}$. In order to distinguish proposals that have similar appearance but belong to different image regions we define the pairwise potential $\psi_{p}$ to be a linear combination of several terms that jointly incorporate color, spatial and temporal information:

$$
\begin{gathered}
\psi_{p}\left(y_{i}, y_{j} ; \mathcal{F}\right)=\left[y_{i} \neq y_{j}\right] \cdot(\underbrace{\omega_{c} k_{c}\left(\mathcal{D}_{c}\left(c_{i}, c_{j}\right)\right)}_{\text {appearance kernel }}+ \\
\underbrace{\omega_{s} k_{s}\left(\mathcal{D}_{s}\left(s_{i}, s_{j}\right)\right)}_{\text {spatial kernel }}+\underbrace{\omega_{p} k_{p}\left(\mathcal{D}_{p}\left(p_{i}, p_{j}\right)\right)}_{\text {trajectory kernel }}+\underbrace{\omega_{t} k_{t}\left(\left|t_{i}-t_{j}\right|\right)}_{\text {temporal kernel }}) .
\end{gathered}
$$

The color appearance $\mathcal{D}_{c}$ is defined in terms of the chisquared kernel $\chi^{2}\left(c_{i}, c_{j}\right)$ where $c_{i}$ and $c_{j}$ are normalized RGB color histograms of proposals $s_{i}$ and $s_{j}$, respectively, with 20 bins per dimension. The spatial relation between any pairs of proposals is defined in terms of the intersectionover-union: $\mathcal{D}_{s}\left(s_{i}, s_{j}\right)=1-\frac{\left|s_{i} \cap s_{j}\right|}{\left|s_{i} \cup s_{j}\right|}$. The last two kernels establish temporal connectivity among proposals, reducing the penalty of assigning different labels to those that are not intersected by the same trajectory or that belong to a different frame. The trajectory kernel exploits that the proposals we use consist of compact sub-regions in the form of superpixels. Let $p_{i} \subset s_{i}$ and $p_{j} \subset s_{j}$ be the set of superpixels that share at least one point-track (computed using [5]) with $s_{j}$ or $s_{i}$, respectively. We define $\mathcal{D}_{p}$ based on the area that is intersected by common trajectories $\mathcal{D}_{p}\left(p_{i}, p_{j}\right)=1-\frac{\left|p_{i} \cup p_{j}\right|}{\left|s_{i} \cup s_{j}\right|}$. In the last term, $t_{i}$ and $t_{j}$ are the corresponding frame numbers of proposals $s_{i}$ and $s_{j}$, which reduces penalty for assigning different labels to proposals that are distant in time. The maximum a posteriori (MAP) labeling of the random field $Y^{*}=\operatorname{argmax}_{Y \in \mathcal{L}} P(Y \mid \mathcal{X}, \mathcal{F})$ minimizing the Gibbs energy $E(Y \mid \mathcal{X}, \mathcal{F})$ produces the segmentation of the video.

To efficiently recover $Y^{*}$ we use the framework of Krähenbühl and Koltun [23], which provides a linear time $O(N)$ algorithm for the inference of $\mathrm{N}$ variables on a fullyconnected graph based on a mean field approximation to the CRF distribution. The efficiency of the method comes with the limitation that the pairwise potential must be expressed as a linear combination of Gaussian kernels having 
the form:

$$
\psi_{p}\left(y_{i}, y_{j}, \mathcal{F}\right)=\mu\left(y_{i}, y_{j}\right) \sum_{m=1}^{K} w_{m} k_{m}\left(\mathbf{f}_{i}, \mathbf{f}_{j}\right)
$$

where each Gaussian kernel defined as:

$$
k_{m}\left(\mathbf{f}_{i}, \mathbf{f}_{j}\right)=\exp \left(-\frac{1}{2}\left(\mathbf{f}_{i}-\mathbf{f}_{j}\right)^{T} \Lambda_{m}\left(\mathbf{f}_{i}-\mathbf{f}_{j}\right)\right) .
$$

We now describe the embedding techniques we employ to project $\mathcal{F}$ into Euclidean space in order to overcome this limitation.

\subsection{Euclidean Embedding}

To enable the use of arbitrary pairwise potentials we seek a new representation of the data in which the $l_{2}$-norm is a good approximation to the distance of the original nonlinear space. In practice, given the original set of features $\mathcal{F}$ we seek a new embedding $\hat{\mathcal{F}}$ into the Euclidean space $\mathbb{R}^{d}$ s.t.:

$$
\mathcal{D}\left(\mathbf{f}_{i}, \mathbf{f}_{j}\right) \approx\left\|\hat{\mathbf{f}}_{i}-\hat{\mathbf{f}}_{j}\right\|_{2} .
$$

Campbell et al. [7] have demonstrated the effectiveness of Landmark Multidimensional Scaling (LMDS) [15] in a context similar to ours. LMDS is an efficient variant of Multidimensional Scaling [13] that uses the Nystrom approximation [3] to reduce the complexity from $O\left(N^{3}\right)$ to $O\left(N m k+m^{3}\right)$ where $N$ is the number of points, $m$ is the number of landmarks and $k$ the dimensionality of the new space. We refer the reader to $[7,36]$ for more details.

We use LMDS to conform the pairwise potential to Eq. (4). We express pairwise potentials $\psi_{p}$ in Eq. (3) as a linear combination of several terms. For better control of the resulting embedding error, we separately embed each of the components. For each $\mathcal{D}_{*}$ term of Eq. (3), we empirically determine the dimensionality of the embedding space from the analysis of their dissimilarity matrix eigenvalues. The resulting pairwise potential conforming to Eq. (4) is:

$$
\begin{aligned}
& \psi_{p}\left(y_{i}, y_{j} ; \hat{\mathcal{F}}\right)=\left[y_{i} \neq y_{j}\right]\left(\omega_{c} k_{c}\left(\hat{c}_{i}, \hat{c}_{j}\right)+\right. \\
& \left.\omega_{s} k_{s}\left(\hat{s}_{i}, \hat{s}_{j}\right)+\omega_{p} k_{p}\left(\hat{p}_{i}, \hat{p}_{j}\right)+\omega_{t} k_{t}\left(t_{i}, t_{j}\right)\right) .
\end{aligned}
$$

The features $\hat{c}, \hat{s}, \hat{p}$ are Euclidean vectors of 10, 20 and 50 dimensions respectively. Note that the temporal term $t$ is already Euclidean, and so it does not require embedding.

\subsection{Segmentation}

The final video segmentation is computed as the sum of the proposals weighted by the conditional probability $P(y=\mathrm{fg} \mid \mathcal{X}, \hat{\mathcal{F}})$ and scaled to range $[0,1]$ on a per-frame basis. As a final post-processing step, we refine the segmentation with a median filter of width 3 applied along the direction of the optical flow [5]. This has the effect of removing temporal instability that arises from different per-frame object proposal configurations. The final segmentation can then be thresholded by $\beta$ to achieve a binary mask.

\begin{tabular}{lc}
\hline Stage & Time (seconds) \\
\hline Optical flow & 113.1 \\
Object Proposals & 55.6 \\
Feature Extraction & 541.7 \\
SVM Classification & 42.7 \\
MDS Embedding & 78.4 \\
CRF Inference & 260.0 \\
\hline Video Segmentation & 1091.5 \\
\hline
\end{tabular}

Table 2. Running time in seconds for each individual stage to segment a video of 75 frames and spatial resolution 960x540.

\section{Implementation Details}

We conducted all experiments on a machine with 2 Intel Xeon $2.20 \mathrm{GHz}$ processors with 8 cores each. The algorithm has been implemented in Python. For the SVM-based pruning we employ the implementation of scikit-learn [33]. Most of the components of our algorithm are parallelizable. Those that are not, such as MDS and the CRF, are relatively efficient. In Table 2 we report the time consumption of each individual component for a sample video of 75 frames and resolution of $960 \times 540$. It takes about 20 minutes to complete the segmentation which is about 16 seconds per frame. The running time performance of our algorithm is comparable to the fastest existing methods such as [17, 32, 37]. The weights of the CRF pairwise potential $\psi_{p}$ of Eq. (3) are specific to the dataset. For FBMS we used $\omega_{c}=1.0$, $\omega_{s}=0.15, \omega_{p}=0.3$ and $\omega_{t}=0.2$, while for SegTrack we reduced the impact of spatial-temporal relationships between proposals setting $\omega_{s}=\omega_{t}=0.01$. The proposal generation step uses 200 seeds, 200 level sets, with the rejection overlap set to 0.95 . The only necessary modification of parameters was a reduction of the number of proposals for the evaluation of the CRF step only (without proposal pruning), which we discuss in detail below. For that experiment, we reduced the number of proposals using 30 seeds, 30 level sets and rejection threshold of 0.88. The parameter $\beta$ that binarizes the final segmentation is set empirically to 0.03 for FBMS and 0.07 for SegTrack.

\section{Results}

We quantitatively evaluate our approach and its components with respect to various state-of-the-art techniques on the Freiburg-Berkeley Motion Segmentation Dataset (FBMS [5]), see Fig. 2. Please also refer to the supplemental material for details.

FBMS Results. The FBMS dataset consists of 59 sequences featuring typical challenges of unconstrained videos such as fast motion, motion blur, occlusions, and object appearance changes. The dataset is split into a training and testing set. Since none of the methods we compare with requires a training phase, we measure performance on both 

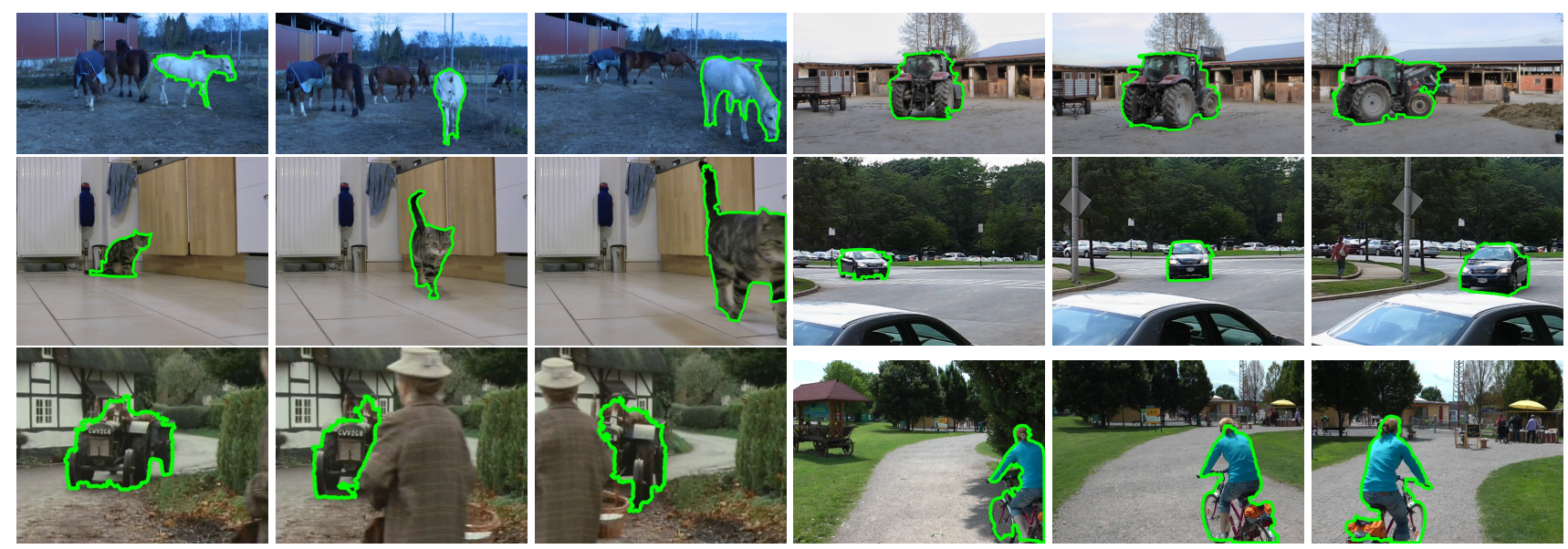

Figure 2. Top to bottom, left to right: qualitative video object segmentation results on six sequences (horses05, farm01, cats01, cars4, marple8 and people5) from the FBMS dataset [5]. Our method demonstrates reasonable segmentation quality for challenging cases, e.g., non-rigid motion and considerable appearance changes (horse05, cats01). The rich set of features of the SVM and the pairwise potentials of the CRF make our method robust to cluttered background (farm1, cars4), while the fully connected graph on which we perform inference provides robustness to partial and full occlusions (marple8). The aggregation of object proposals is also effective for complex, multi-colored objects (people05).

sets. Due to running-time and memory constraints of the prior approaches that we compare to, we limit the length of the videos to 75 frames. For the purpose of testing segmentation quality in the presence of fast motion we temporally subsample frames from videos exhibiting slow motion. We report the speed-up factor of each sequence in the supplementary material. In videos that have multiple objects we manually selected the one with dominant motion. Similar to previous works $[17,27]$ we measure the segmentation quality in terms of intersection-over-union, which is invariant to image resolution and to the size of the foreground object.

We compare our method (FCP) with several recent stateof-the-art approaches [17, 32, 37,44]. These methods have been selected based on their quality of results, underlying approaches, and availability of their source code. SeamSeg (SEA, [37]), seeks connected paths of low energy to track the object boundaries. Zhang et al. (DAG, [44]) integrate objectness and appearance similarity in a directly acyclic graph whose shortest-path corresponds to a video segmentation. Under the assumption that the object moves differently from the background Papazoglou and Ferrari (FST, [32]) find the closed motion boundary and propagate the initial estimate using a spatio-temporal optimization. Finally, Faktor and Irani (NLC, [17]) consolidate an initial foreground estimate based on saliency using a Markov chain.

Our method and SEA are semi-supervised while the others are unsupervised. For a more informative and fairer comparison, we therefore removed any of the videos from the comparison in Table 3, for which at least one of these unsupervised methods did not detect the object.We report detailed sequence evaluation for the test set and the average for the training set. Please also refer to the supplementary material for a more detailed evaluation. We separately evaluate the steps of our algorithm: SVM only, CRF only, and the full approach FCP. Corresponding precision, recall, and f-measure plots are shown in Fig. 3. As discussed in the implementation section, in the CRF experiment we modified the parameters generating object proposals to produce roughly the same number of proposals that are retained during the pruning step.

Results in Table 3 demonstrates that our method consistently produces a good segmentation yielding roughly a $10 \%$ improvement over the current state-of-the-art in terms of average performance. The importance of combining both the SVM and CRF steps is also apparent.

Limitations. Our approach is designed to work with real-world video sequences with fast object motion, and occlusions. In particular, since our method is based on object proposals, it requires a sufficiently high video resolution such that the computation of proposals using existing techniques produces meaningful results. This becomes clear in Table 4, when running our approach on lower resolution video such as the SegTrack benchmark ${ }^{1}[26,32]$. We additionally compare with (TMF, [27]), (KEY, [26]) and (HVS, [19]). In this dataset, very few proposals overlap with the foreground object due to the limited image resolution (highest is $414 \times 320$ ) and the small size of the objects, so our approach, which is based on aggregating multiple object hypothesis works less well. For example the training set of the birdfall video has a ratio of 1:4000 foreground and background proposals, with only 13 proposals on the object. The lack of positive samples weakens the self-training of

\footnotetext{
${ }^{1}$ In accordance with prior works we do not evaluate 'penguin'
} 

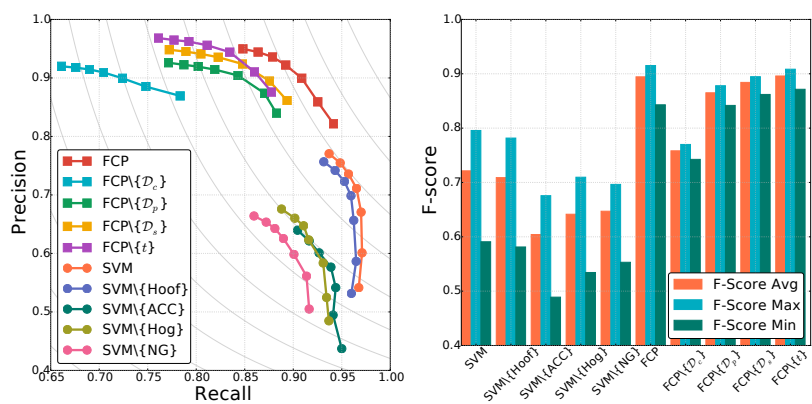

Figure 3. Left: Precision-Recall curves and $F$-score isolines $\left(\beta^{2}=\right.$ 0.3 ) for the SVM and CRF classification of object proposals into foreground and background, obtained by varying the minimum amount of overlap $\tau$ required for a proposal to be considered foreground. The SVM classification (SVM) is less precise but has better recall, preventing the removal of foreground proposals, with which the CRF can perform the final classification (FCP). The plot also evaluates the individual importance of the SVM features of Table 1 and the CRF potentials of Eq. (3) in terms of the resulting loss if they were removed during the classification. Right: Average, maximum and minimum F-score. Our solution FCP outperforms the SVM only classification. Note that the best scores, respectively SVM and FCP, are obtained when all features and potentials are employed.

the SVM and, as consequence, the effectiveness of the CRF is severely limited. While some of the results are comparable with other approaches, these limitations are the reason why our method performs significantly better on the FBMS dataset.

\section{Conclusion}

We presented a novel approach to segment objects in unconstrained videos, which provides state-of-the-art performance on challenging video data. In general we believe that, due to the constant increase in terms of video resolution and quality, even more complex benchmarks than FBMS are required to provide real-world application scenarios for evaluating video segmentation algorithms. However, methods based on object proposals appear to be a great candidate for addressing the computational challenges arising from higher resolution video data, since the use of proposals greatly reduces computational complexity, allowing us to employ a fully connected CRF over a complete video sequence. A similar, fully connected formulation at the pixel level would be infeasible. There exist several further opportunities for followup work. For example, to improve the final segmentation accuracy, it would be interesting to investigate approaches to combine the prediction of the CRF in a more principled manner (e.g., incorporating higher-order potentials), or to employ bilateral filtering techniques to refine the proposal-based segmentation to the pixel level.

\begin{tabular}{cccccccc}
\hline Sequence & FCP & CRF & SVM & SEA & FST & DAG & NLC \\
\hline cars1 & 0.69 & 0.80 & 0.68 & $\mathbf{( 0 . 8 3 )}$ & 0.82 & 0.10 & 0.27 \\
cats01 & $\mathbf{( 0 . 8 3 )}$ & 0.68 & 0.76 & 0.62 & 0.80 & 0.34 & 0.71 \\
cats03 & 0.39 & 0.00 & 0.11 & 0.17 & $\mathbf{( 0 . 5 3 )}$ & 0.32 & 0.12 \\
dogs01 & 0.55 & 0.22 & 0.39 & 0.38 & 0.53 & $\mathbf{( 0 . 5 6 )}$ & 0.54 \\
goats01 & 0.82 & $\mathbf{( 0 . 8 4 )}$ & 0.78 & 0.53 & $\mathbf{( 0 . 8 4 )}$ & 0.79 & 0.58 \\
horses05 & $\mathbf{( 0 . 7 7 )}$ & 0.66 & 0.47 & 0.69 & 0.34 & 0.44 & 0.38 \\
lion01 & $\mathbf{( 0 . 8 4 )}$ & 0.74 & 0.80 & 0.73 & 0.80 & 0.77 & 0.67 \\
marple2 & 0.59 & 0.57 & 0.71 & $\mathbf{( 0 . 7 8 )}$ & 0.65 & 0.56 & 0.60 \\
marple4 & $\mathbf{( 0 . 8 8 )}$ & 0.73 & 0.87 & 0.69 & 0.15 & 0.45 & 0.19 \\
marple6 & 0.77 & 0.64 & 0.77 & $\mathbf{( 0 . 8 6 )}$ & 0.24 & 0.18 & 0.48 \\
people1 & 0.68 & 0.64 & 0.22 & 0.58 & 0.54 & 0.69 & $\mathbf{( 0 . 8 5 )}$ \\
people2 & 0.81 & 0.78 & 0.76 & 0.77 & $\mathbf{( 0 . 9 2 )}$ & 0.48 & 0.77 \\
rabbits02 & 0.66 & 0.11 & 0.33 & 0.42 & 0.65 & 0.32 & $\mathbf{( 0 . 7 1 )}$ \\
rabbits03 & 0.43 & 0.40 & 0.23 & 0.42 & 0.41 & 0.22 & $\mathbf{( 0 . 4 4 )}$ \\
rabbits04 & 0.29 & 0.00 & 0.12 & 0.23 & $\mathbf{( 0 . 3 8 )}$ & 0.12 & 0.20 \\
tennis & 0.48 & 0.27 & 0.41 & 0.55 & 0.30 & 0.51 & $\mathbf{( 0 . 6 4 )}$ \\
\hline Avg. Test & $\mathbf{( 0 . 6 5 )}$ & 0.51 & 0.53 & 0.58 & 0.56 & 0.43 & 0.51 \\
Avg. Training & $\mathbf{( 0 . 7 7 )}$ & 0.62 & 0.61 & 0.71 & 0.68 & 0.60 & 0.56 \\
\hline
\end{tabular}

Table 3. Intersection-over-union comparisons on a subset of the FBMS dataset. The columns SVM and CRF correspond to the results obtained using either only our SVM-based classification, or only our CRF-based labeling, respectively. Our full approach (FCP) is generally (close to) the best performing one (highlighted in bold), and achieves the highest average values of all methods.

\begin{tabular}{cccccccc}
\hline Sequence & FCP & NLC & FST & DAG & TMF & KEY & HVS \\
\hline birdfall & 0.25 & $\mathbf{( 0 . 7 4 )}$ & 0.59 & 0.71 & 0.62 & 0.49 & 0.57 \\
cheetah & 0.49 & $\mathbf{( 0 . 6 9 )}$ & 0.28 & 0.4 & 0.37 & 0.44 & 0.19 \\
girl & 0.54 & $\mathbf{( 0 . 9 1 )}$ & 0.73 & 0.82 & 0.89 & 0.88 & 0.32 \\
monkeydog & 0.64 & 0.78 & $\mathbf{( 0 . 7 9 )}$ & 0.75 & 0.71 & 0.74 & 0.68 \\
parachute & 0.91 & 0.94 & 0.91 & 0.94 & 0.93 & $\mathbf{( 0 . 9 6 )}$ & 0.69 \\
\hline Average & 0.57 & $\mathbf{( 0 . 8 1 )}$ & 0.66 & 0.72 & 0.70 & 0.70 & 0.49 \\
\hline
\end{tabular}

Table 4. Intersection-over-union computed on the SegTrack dataset. On low resolution video, there are insufficient foreground proposals generated for our method to work well.

Acknowledgements This work was supported by an SNF award (200021_143598).

\section{References}

[1] P. A. Arbeláez, J. Pont-Tuset, J. T. Barron, F. Marqués, and J. Malik. Multiscale combinatorial grouping. In Proc. CVPR, 2014. 2

[2] D. Banica, A. Agape, A. Ion, and C. Sminchisescu. Video object segmentation by salient segment chain composition. In The IEEE International Conference on Computer Vision (ICCV) Workshops, December 2013. 2

[3] S. Belongie, C. Fowlkes, F. R. K. Chung, and J. Malik. Spectral partitioning with indefinite kernels using the nyström extension. In Proc. ECCV, 2002. 5

[4] W. Brendel and S. Todorovic. Video object segmentation by tracking regions. In Proc. ICCV, 2009. 2

[5] T. Brox and J. Malik. Object segmentation by long term analysis of point trajectories. In Proc. ECCV, 2010. 1, 2, 4, 5,6 
[6] S. Brutzer, B. Höferlin, and G. Heidemann. Evaluation of background subtraction techniques for video surveillance. In Proc. CVPR, 2011. 2

[7] N. D. F. Campbell, K. Subr, and J. Kautz. Fully-connected crfs with non-parametric pairwise potential. In Proc. CVPR, 2013. 5

[8] J. Carreira, F. Li, and C. Sminchisescu. Object recognition by sequential figure-ground ranking. IJCV , 98(3):243-262, 2012. 2

[9] J. Carreira and C. Sminchisescu. CPMC: automatic object segmentation using constrained parametric min-cuts. IEEE TPAMI, 34(7):1312-1328, 2012. 2

[10] R. Chaudhry, A. Ravichandran, G. D. Hager, and R. Vidal. Histograms of oriented optical flow and binet-cauchy kernels on nonlinear dynamical systems for the recognition of human actions. In Proc. CVPR, 2009. 3

[11] M. Cheng, Z. Zhang, W. Lin, and P. H. S. Torr. BING: binarized normed gradients for objectness estimation at 300fps. In Proc. CVPR, 2014. 3

[12] S.-C. S. Cheung and C. Kamath. Robust techniques for background subtraction in urban traffic video. In Visual Communications and Image Processing, volume 5308, pages 881892, 2004. 2

[13] T. Cox and M. Cox. Multidimensional Scaling. Chapman \& Hall, London, 1994. 5

[14] A. Criminisi, G. Cross, A. Blake, and V. Kolmogorov. Bilayer segmentation of live video. In Proc. CVPR, 2006. 2

[15] V. de Silva and J. B. Tenenbaum. Global versus local methods in nonlinear dimensionality reduction. In S. Becker, S. Thrun, and K. Obermayer, editors, Proc. NIPS, 2002. 5

[16] I. Endres and D. Hoiem. Category-independent object proposals with diverse ranking. IEEE TPAMI, 36(2):222-234, 2014. 2, 3

[17] A. Faktor and M. Irani. Video segmentation by non-local consensus voting. In Proc. BMVC, 2014. 2, 5, 6

[18] R. B. Girshick, J. Donahue, T. Darrell, and J. Malik. Rich feature hierarchies for accurate object detection and semantic segmentation. In Proc. CVPR, 2014. 2

[19] M. Grundmann, V. Kwatra, M. Han, and I. A. Essa. Efficient hierarchical graph-based video segmentation. In Proc. CVPR, 2010. 2, 6

[20] E. Hayman and J. Eklundh. Statistical background subtraction for a mobile observer. In Proc. ICCV, 2003. 2

[21] S. Hickson, S. Birchfield, I. A. Essa, and H. I. Christensen. Efficient hierarchical graph-based segmentation of RGBD videos. In Proc. CVPR, 2014. 2

[22] M. Irani and P. Anandan. A unified approach to moving object detection in $2 \mathrm{~d}$ and $3 \mathrm{~d}$ scenes. IEEE TPAMI, 20(6):577589, 1998. 2

[23] P. Krähenbühl and V. Koltun. Efficient inference in fully connected crfs with gaussian edge potentials. In Proc. NIPS, 2011. 4

[24] P. Krähenbühl and V. Koltun. Geodesic object proposals. In Proc. ECCV, 2014. 2, 3

[25] J. D. Lafferty, A. McCallum, and F. C. N. Pereira. Conditional random fields: Probabilistic models for segmenting and labeling sequence data. In ICML, 2001.
[26] Y. J. Lee, J. Kim, and K. Grauman. Key-segments for video object segmentation. In Proc. ICCV, 2011. 2, 3, 6

[27] F. Li, T. Kim, A. Humayun, D. Tsai, and J. M. Rehg. Video segmentation by tracking many figure-ground segments. In Proc. ICCV, 2013. 1, 2, 6

[28] T. Lin, M. Maire, S. Belongie, J. Hays, P. Perona, D. Ramanan, P. Dollár, and C. L. Zitnick. Microsoft COCO: common objects in context. In Proc. ECCV, 2014. 2

[29] D. G. Lowe. Distinctive image features from scale-invariant keypoints. IJCV, 60(2):91-110, 2004. 3

[30] T. Ma and L. J. Latecki. Maximum weight cliques with mutex constraints for video object segmentation. In Proc. CVPR, 2012. 1, 2

[31] P. Ochs and T. Brox. Object segmentation in video: A hierarchical variational approach for turning point trajectories into dense regions. In Proc. ICCV, 2011. 2

[32] A. Papazoglou and V. Ferrari. Fast object segmentation in unconstrained video. In Proc. ICCV, 2013. 2, 5, 6

[33] F. Pedregosa, G. Varoquaux, A. Gramfort, V. Michel, B. Thirion, O. Grisel, M. Blondel, P. Prettenhofer, R. Weiss, V. Dubourg, J. Vanderplas, A. Passos, D. Cournapeau, M. Brucher, M. Perrot, and E. Duchesnay. Scikit-learn: Machine learning in Python. Journal of Machine Learning Research, 12:2825-2830, 2011. 5

[34] F. Perazzi, P. Krähenbühl, Y. Pritch, and A. Hornung. Saliency filters: Contrast based filtering for salient region detection. In Proc. CVPR, 2012. 3

[35] J. C. Platt. Probabilistic outputs for support vector machines and comparisons to regularized likelihood methods. In Advances in large margin classifiers, pages 61-74, 1999. 3

[36] J. C. Platt. Fastmap, metricmap, and landmark mds are all nystrom algorithms. In Proc. Workshop on Artificial Intelligence and Statistics, pages 261-268, 2005. 5

[37] S. A. Ramakanth and R. V. Babu. Seamseg: Video object segmentation using patch seams. In Proc. CVPR, 2014. 2, 5, 6

[38] Y. Ren, C. Chua, and Y. Ho. Statistical background modeling for non-stationary camera. Pattern Recognition Letters, 24(1-3):183-196, 2003. 2

[39] C. Rother, V. Kolmogorov, and A. Blake. "grabcut": interactive foreground extraction using iterated graph cuts. $A C M$ Trans. Graph., 23(3):309-314, 2004. 2

[40] M. Rubinstein, A. Shamir, and S. Avidan. Improved seam carving for video retargeting. ACM Trans. Graph., 27(3), 2008. 2

[41] D. Varas and F. Marqués. Region-based particle filter for video object segmentation. In Proc. CVPR, 2014. 2

[42] B. X. Wang and N. Japkowicz. Boosting support vector machines for imbalanced data sets. In Proc. ISMIS, 2008. 3

[43] C. Xu and J. J. Corso. Evaluation of super-voxel methods for early video processing. In Proc. CVPR, 2012. 2

[44] D. Zhang, O. Javed, and M. Shah. Video object segmentation through spatially accurate and temporally dense extraction of primary object regions. In Proc. CVPR, 2013. 1, 2, 6 